\section{Next-generation sequencing to estimate the prevalence of a great unknown: McArdle disease}

To the Editor: McArdle disease is caused by inherited deficiency of myophosphorylase, the PYGM-encoded enzyme that catalyzes the breakdown of glycogen into glucose-1-phosphate in muscle fibers. De Castro et al. ${ }^{1}$ recently suggested that the currently accepted disease prevalence in North Americans of European descent $(\sim 1 / 100,000)$ is an underestimate. By their calculations, the actual prevalence would be more than twice as high in this population, or even more than three times higher, compared with the data we recently reported for Caucasian Spaniards $(\sim 1 / 167,000))^{2}$ Their predictions were based on a novel approach. Instead of studying people with diagnosed/ suspected McArdle disease, next-generation sequencing of PYGM was performed in people with no primary muscle disease. Their timely study indicates that many patients remain undiagnosed.

We have been working in different areas of this disease for more than 10 years, including the generation of the Spanish patients' registry. In January 2010, the number of diagnosed cases was 239. ${ }^{2}$ All were homozygous or compound heterozygous for documented PYGM mutations. Most, if not all, showed a main disease feature associated with the inability to use an important fuel, glycogen, for muscle contractions and had intolerance to dynamic exercise (brisk walking, running, swimming) or isometric muscle contractions (carrying weights), usually in the form of acute crises of contractures, undue fatigue, and muscle pain. Of note, the intensity/type of the exercise stimuli triggering such crises varied considerably between individuals (see below). Virtually all had high baseline levels of serum creatine kinase activity (an indicator of muscle damage), and most reported the "second wind" phenomenon, that is, the ability to resume dynamic exercise if they took a brief rest upon the appearance of premature fatigue and muscle pain. Yet, only 5 years later, we have identified $\sim 100$ new patients on the basis of Sanger PYGM sequencing, which translates to a prevalence of $\sim 1 / 140,000$. Thus, we concur with De Castro et al. ${ }^{1}$ that current estimates of the disease prevalence are low. However, based on our experience, we think that this underestimation is due not to the existence of undiscovered disease phenotypes or to the nonmonogenic nature of this disorder (with other genes awaiting to be found), as De Castro et al. ${ }^{1}$ suggest, but, rather, to its own particularities. Some potential patients might not be referred to genetic diagnosis simply because McArdle disease is a great unknown.
In our experience, symptoms start during childhood, often in physical education classes. However, diagnosis was delayed until later ages in $96 \%$ of Spanish patients. ${ }^{2}$ Children seldom perform extended sessions of dynamic exercise because shorter, more "explosive," bouts of activity (jumping, sprinting) are more natural at earlier ages. Thus, they are less likely to report the second-wind phenomenon, which is considered a pathognomonic feature in adult patients. Simple recognition of the fact that children are not small adults and therefore may manifest exercise intolerance differently might result in referring more children to timely genetic diagnosis in the near future and recommendations of adoption of an active lifestyle from early life (see below).

We have also noted that $\sim 50 \%$ of patients do not report episodes of dark urine due to severe rhabdomyolysis, even though such episodes have frequently been viewed as a hallmark of the disease. In fact, there is large individual variability in clinical severity and some patients have intolerance only to strenuous exercise, e.g., sports activities. ${ }^{2}$ Thus, with approximately one-third of adult Westerners being currently inactive-not even walking $150 \mathrm{~min} /$ week and most not participating in any sport-it is not unreasonable to suspect that some cases remain unnoticed.

We do not fully agree with another conclusion of the study by De Castro et al., ${ }^{1}$ namely, that some of the genetic variants considered pathogenic might actually be "benign," at least from what is known of the disease. In a recent review, we found that 147 different mutations (91\% exonic and $9 \%$ intronic) have been reported in the PYGM gene. ${ }^{3}$ The most frequent mutations are missense $(50 \%)$, followed by deletions $(18 \%)$, nonsense mutations (13\%), and mutations affecting RNA splicing (11\%). Most have been shown to have functional consequences, and many result in no gene transcript levels owing to a homeostatic mechanism, the so-called non-sense-mediated decay, which regulates the quality of the transcripts inside each cell by degrading those that contain premature termination codons. The action of non-sense-mediated decay explains, at least partly, why patients typically present null myophosphorylase activity in muscle biopsy samples, except for three cases reported in the literature (two North American patients and one Japanese patient $^{3}$ ), e.g., $69 \%$ of all registered Spanish patients and 100\% of those for whom muscle biopsy sample were analyzed. ${ }^{2}$ Furthermore, there is no PYGM genotype-phenotype correlation despite the large heterogeneity commonly reported both in mutation types (e.g., 108 different PYGM genotypes in the Spanish registry) and disease severity. Some patients ( $\sim 8 \%$ in the Spanish registry) are virtually asymptomatic during activities of daily living but many more ( 50\%) are limited even during daily life. In addition, the commonest nonsense p.R50X (or p.Arg50*) mutation (resulting in non-sense-mediated decay) is also present among the less affected patients. Other factors, 
notably patients' physical activity habits, explain the individual variability in the phenotype manifestation of a common muscle defect as total myophosphorylase deficiency. Patients who exercise regularly-ideally under careful professional supervision-are much more likely to be less affected over the years. The beneficial muscle biological adaptations to regular exercise (increased oxidative capacity or muscle mass and power, among others) are likely to compensate, at least during nonstrenuous activities, for the inherited blockade in glycogenolysis. ${ }^{2}$

Finally, the next-generation sequencing approach proposed by De Castro et al. ${ }^{1}$ is also important in that it might allow identification of PYGM polymorphisms, which have been largely uncovered in this disease, as we recently said in a review. ${ }^{3}$ Publication of the polymorphisms discovered by the authors is potentially of great utility and in fact acknowledged by those who treat McArdle patients.

\section{DISCLOSURE}

The authors declare no conflict of interest.

\section{Response to Nogales-Gadea et al.}

To the Editor: In their letter, Nogales-Gadea et al. ${ }^{1}$ present a thoughtful analysis of several points from our recently published paper entitled "Determining the Prevalence of McArdle Disease From Gene Frequency by Analysis of Next-Generation Sequencing Data." The main thrust of our paper is that McArdle disease is more common than currently appreciated. Overall, Nogales-Gadea et al. ${ }^{1}$ agreed with our conclusion, if not taking it even further, but they differed on the weighting of some of our explanations for why it may be underrecognized. These interesting and important questions are worthy of further discussion.

Nogales-Gadea et al. ${ }^{1}$ agree that one explanation for our finding is that an unknown, but significant, proportion of patients are undiagnosed by virtue of having mild or no symptoms, which could be due to the intrinsic biology of the disorder or because the level of physical activity of many individuals with biallelic mutations in PYGM is not sufficient to trigger development of recognizable manifestations. We fully agree with this and believe that the penetrance and expressivity of McArdle disease are complex questions that deserve further study.

In our paper, we showed several alternative calculations based on carrier frequency that led us to estimate that prevalence of McArdle disease was $1 / 7,650$ to $1 / 80,478$, which is lower than previously estimated $(1 / 100,000)$. We thought that the high end of this range $(1 / 7,650)$ was unlikely and suggested as one possible explanation that some variants reported as pathogenic may actually be benign, consistent
Gisela Nogales-Gadea, $P h D^{1}$, Tomàs Pinós, $P h D^{2,3}$, Antoni L. Andreu, MD, PhD ${ }^{2,3}$, Miguel A. Martín, $P h D^{3-5}$, Joaquin Arenas, $P h D^{4,5}$, and Alejandro Lucia, $M D, P h D^{5,6}$

${ }^{1}$ Department of Neurosciences, Institut d'Investigació en Ciències de la Salut Germans Trias i Pujol I Campus Can Ruti, Universitat Autònoma de Barcelona, Badalona, Spain; ${ }^{2}$ Departament de Patologia Mitocondrial i Neuromuscular, Hospital Universitari Vall d'Hebron, Institut de Recerca (VHIR), Universitat Autónoma de Barcelona, Barcelona, Spain; ${ }^{3}$ Centre for Biomedical Network Research on Rare Diseases, Instituto de Salud Carlos III, Madrid, Spain; ${ }^{4}$ Laboratorio de Enfermedades Mitocondriales y Neuromusculares. Hospital 12 de Octubre, Madrid, Spain; ${ }^{5}$ Instituto de Investigación Hospital 12 de Octubre (i+12), Madrid, Spain; ${ }^{6}$ Universidad Europea, Madrid, Spain. Correspondence: Gisela Nogales-Gadea (gnogales@igtp.cat)

\section{REFERENCES}

1. De Castro M, Johnston J, Biesecker L. Determining the prevalence of McArdle disease from gene frequency by analysis of next-generation sequencing data. Genet Med; e-pub ahead of print 5 March 2015.

2. Lucia A, Ruiz JR, Santalla A, et al. Genotypic and phenotypic features of McArdle disease: insights from the Spanish national registry. J Neuro/ Neurosurg Psychiatr 2012;83:322-328.

3. Nogales-Gadea G, Brull A, Santalla A, et al. Mcardle disease: update of reported mutations and polymorphisms in the Pygm gene. Hum Mutat; e-pub ahead of print 25 April 2015.

doi:10.1038/gim.2015.76

with the findings of Bell et al. ${ }^{3}$ Nogales-Gadea et al. ${ }^{1}$ questioned this hypothesis. They have very recently reviewed PYGM mutations in McArdle disease, and they generously shared a draft manuscript with us to support our writing of this response. ${ }^{4}$ We repeated our calculations, including all variants reported as pathogenic in their manuscript, using the European-American subset of the National Heart, Lung, and Blood Institute's Exome Sequencing Project data set. The result was a prevalence estimate for McArdle disease of about $1 / 6,000$, more common than even the highest estimate in our paper. More accurately, we should say that this is the predicted prevalence of individuals who harbor biallelic pathogenic mutations in PYGM. Assuming this to be true, one must then explain why the prevalence of the McArdle phenotype appears to be so much lower than this. We and Nogales-Gadea et al. ${ }^{1}$ invoke various explanations that relate to the penetrance and expressivity of this trait and believe that it can be considered a gene-environment interaction, for which sustained energetic exercise is the environmental exposure or trigger. The authors are surely correct when they suggest that sedentary individuals have a lower probability of manifesting symptoms that might lead to a diagnosis of McArdle disease. Another potential explanation is the existence of modifiers. These could be acting in cis, analogous to the c.350G >A p.Arg117His and poly$\mathrm{T}$ tract (ref. 5) or in trans, analogous to variants in DCTN4 (ref. 6), both of which modify the phenotype associated with mutations in CFTR.

We also agree with Nogales-Gadea et al. ${ }^{1}$ that next-generation sequencing highlights other potentially interesting issues. We did not address in our manuscript the observation that European-American data sets contain a number of apparently null PYGM variants that have not been observed in patients with disease. We have demonstrated that-at least in autosomal 